\title{
WIENER MEASURE IN A SPACE OF FUNCTIONS OF TWO VARIABLES $\left({ }^{1}\right)$
}

BY

J. YEH

1. Introduction. The Wiener space $C_{2}$ of functions of two variables is the collection of continuous functions $\{x(t, \tau)\}$ on the unit square $0 \leqq t, \tau \leqq 1$ satisfying $x(0, \tau)=x(t, 0)=0$. Integration on this space was first introduced by T. Kitagawa in his Analysis of variance applied to function spaces [2]. There he defined the Wiener integral for functionals on $C_{2}$ of the type $H\left(x\left(t_{1}, \tau_{1}\right), \cdots, x\left(t_{r}, \tau_{s}\right)\right)$ where $H\left(\eta_{11}, \cdots, \eta_{r s}\right)$ is a function of $r s$ real variables $\left\{\eta_{h k}\right\}(h=1,2, \cdots, r, k=1,2, \cdots, s)$ and $\left\{t_{h}\right\},\left\{\tau_{k}\right\}$ are preassigned division points of the unit intervals $0 \leqq t \leqq 1,0 \leqq \tau \leqq 1$ satisfying $0=t_{0} \leqq t_{1}$ $\leqq \cdots \leqq t_{r} \leqq t_{r+1}=1,0=\tau_{0} \leqq \tau_{1} \leqq \cdots \leqq \tau_{s} \leqq \tau_{s+1}=1$. The Wiener integral for this class of functionals was defined to be

$$
\begin{aligned}
\int_{C_{2}}^{w} H\left(x\left(t_{1}, \tau_{1}\right), \cdots\right. & \left., x\left(t_{r}, \tau_{s}\right)\right) d_{w} x \\
& =\int_{-\infty}^{\infty} \cdots \int_{-\infty}^{\infty} H\left(\eta_{11}, \cdots, \eta_{r s}\right) \prod_{h=1}^{r} \prod_{k=1}^{s} p\left(\Delta_{h, k}\right) d \eta_{11} \cdots d \eta_{r, s}
\end{aligned}
$$

where

$$
\begin{aligned}
p\left(\Delta_{h, k}\right)= & \frac{1}{\left[\pi\left(t_{h}-t_{h-1}\right)\left(\tau_{k}-\tau_{k-1}\right)\right]^{1 / 2}} \\
& \cdot \exp \left\{\frac{-\left(\eta_{h, k}-\eta_{h, k-1}-\eta_{h-1, k}+\eta_{h-1, k-1}\right)^{2}}{\left(t_{h}-t_{h-1}\right)\left(\tau_{k}-\tau_{k-1}\right)}\right\} .
\end{aligned}
$$

He gave no proof that this definition of Wiener integral could be extended to more general functionals or that it had the properties of a Lebesgue integral.

It is the purpose of the present paper to define an interval class (or semialgebra) of subsets of the $C_{2}$ space, a set function on this interval class and then prove that this set function is indeed a measure defined on the interval class. Once this is accomplished the measure can be extended to a measure on the Carathéodory extension of the interval class in the usual way. With this Carathéodory extension measureable functionals on $C_{2}$ may be defined

Received by the editors May 11, 1959.

(1) The present proof for the countable additivity of the Wiener measure is a generalization of an unpublished proof for the one-dimensional case given in lecture notes by R. H. Cameron. The author wishes to thank Professor Cameron for his advice in the writing of this paper. 
and their integration on $C_{2}$ may be considered. This not only makes integration of more general functionals than those in [2] possible but it also puts the subject on a firm logical foundation instead of mere optimism.

The definitions of the interval class and the Wiener measure are given by (2.1), (2.2), (2.3).

2. Definition of Wiener measure for $C_{2}$. For the convenience we shall use different notations from those in [2]. Each element of $C_{2}$ will be written $f(x, y)$. Then $C_{2}$ is the collection of real valued continuous functions $f(x, y)$ on the domain $0 \leqq x, y \leqq 1$ satisfying $f(0, y)=f(x, 0)=0$. A pair of real numbers $(x, y)$ with $0 \leqq x, y \leqq 1$ is made to correspond to a point in the unit square of the $x y$-plane in the usual way. If $P$ is a point in this square with coordinates $(x, y)$, we will write $f(P)$ for $f(x, y)$.

A class $\mathcal{J}$ of subsets of a space $S$ is called an interval class if the following three conditions are satisfied:

1. If $I_{1}, I_{2} \in \mathcal{J}$, then $I_{1} \cap I_{2} \in \mathcal{J}$.

2. $\varnothing, S \in \mathcal{d}$.

3. If $I \in \mathcal{J}$, then there exists a finite sequence of elements of $\mathcal{J}, I_{0}, I_{1}, \cdots$, $I_{n}$ such that $I=I_{0} \subset I_{1} \subset \cdots \subset I_{n}=S$ and $I_{j}-I_{j-1} \in \mathcal{J}(n=1,2, \cdots, n)$.

A nonempty class $\mathcal{F}$ of subsets of a space $S$ is called a field of sets if the union of any two elements of $\mathcal{F}$ and the complement of every element of $\mathcal{F}$ are also elements of $\mathfrak{F}$. If further the union of any countable collection of elements of $\mathcal{F}$ belongs to $\mathcal{F}$ then $\mathcal{F}$ is called a Borel field of sets. In particular a field of sets is an interval class.

An extended positive valued set function $m(I)$ on an interval class $g$ is called a measure if it is countably additive and $m(\varnothing)=0$. Here by the extended positive number system we mean all the non-negative numbers and $+\infty$.

To define an interval class $\mathcal{J}$ of subsets of $C_{2}$, let two finite sequences of numbers $0=x_{0}<x_{1}<\cdots<x_{m} \leqq 1,0=y_{0}<y_{1}<\cdots<y_{n} \leqq 1$ and a Lebesgue measurable set $E$ of the $m n$-dimensional Euclidean space $R_{m n}$ be given. Let a subset $I$ of $C_{2}$ corresponding to the two sequences and the measurable set $E$ be defined by

$$
\begin{aligned}
I\left\{x_{1}, \cdots, x_{m}, y_{1}, \cdots, y_{n}, E\right\} & \\
= & \left\{f \in C_{2} ;\left[f\left(x_{1}, y_{1}\right), \cdots, f\left(x_{m}, y_{n}\right)\right] \in E\right\} .
\end{aligned}
$$

The $m n$ points in the unit square, $\left(x_{1}, y_{1}\right), \cdots,\left(x_{m}, y_{n}\right)$, and the set $E$ will be called restriction points and restricting set of the set $I$. For a given collection of $m n$ restriction points and a given restricting set a subset $I$ of $C_{2}$ is uniquely determined by (2.1), but the converse is not true. For instance for the subset $I$ defined by (2.1) we may throw in a few more partition points in the intervals $0 \leqq x \leqq 1,0 \leqq y \leqq 1$, so that there are $p$ and $q$ additional partition points on the unit $x$-interval and the unit $y$-interval respectively; let the restriction at each of the additional restriction points $\left(x_{i}, y_{j}\right)$ be the trivial 
restriction $-\infty<f\left(x_{i}, y_{j}\right)<+\infty$ and let the restriction set be $E \otimes R_{p n+q m+p q}$, a Lebesgue measurable set in $R_{(m+p)(n+q)}$. The subset of $C_{2}$ defined with the $(m+p)(n+q)$ restriction points and the restricting set $R_{(m+p)(n+q)}$ is identical with the one defined by (2.1).

Now let $\mathcal{J}$ be the collection of subsets of $C_{2}$ of the type (2.1). To show that $\mathcal{J}$ is an interval class, or even more that $\mathcal{J}$ is a field of sets, we observe the following. When we have two sets $I_{1}\left\{x_{11}, \cdots, x_{1, m_{1}}, y_{11}, \cdots, y_{1, n_{1}}, E_{1}\right\}$ and $I_{2}\left\{x_{21}, \cdots, x_{2, m_{2}}, y_{21}, \cdots, y_{2, n_{2}}, E_{2}\right\}$ we can make them comparable in terms of their restricting sets by using the Cartesian product of the partition points $\left\{x_{11}, \cdots, x_{1, m_{1}}\right\} \cup\left\{x_{21}, \cdots, x_{2, m_{2}}\right\}$ and $\left\{y_{11}, \cdots, y_{1, n_{1}}\right\}$ $\cup\left\{y_{21}, \cdots, y_{2, n_{2}}\right\}$ as their restriction points by means of adding trivial restriction at each newly introduced restriction point and extending their restriction sets to two Lebesgue measurable sets $E_{1}^{\prime}$ and $E_{2}^{\prime}$ of the same Euclidean space. Then in comparing $I_{1}$ and $I_{2}$ we only have to compare $E_{1}^{\prime}$ and $E_{2}^{\prime}$. This procedure can be generalized for any finite collection of sets $I_{1}, I_{2}, \cdots, I_{l}$. If restricting sets $E_{1}, E_{2}, \cdots, E_{l}$ are extended by the above procedure to $E_{1}^{\prime}, E_{2}^{\prime}, \cdots, E_{l}^{\prime}$ all in the same Euclidean space then $I_{3}$ $=I_{1} \cup I_{2}$ if and only if $E_{3}^{\prime}=E_{1}^{\prime} \cup E_{2}^{\prime}, I_{2}=\left(I_{1}\right)^{c}$ if and only if $E_{2}^{\prime}=\left(E_{1}^{\prime}\right)^{c}$ and $I_{3}=I_{1} \cap I_{2}$ if and only if $E_{3}^{\prime}=E_{1}^{\prime} \cap E_{2}^{\prime}$. From the first two of the above three propositions and from the fact that the collection of Lebesgue measurable sets is a Borel field of sets, it follows that $g$ is a field of sets and in particular an interval class.

Now if an interval $I \in \mathcal{J}$ is defined by $(2.1)$, then $m(I)$ is given by

$$
m(I)=\int_{E}^{(m n)} \int W\left\{x_{1}, \cdots, x_{m}, y_{1}, \cdots, y_{n}\right\} d u_{11} \cdots d u_{m n}
$$

where

$$
\begin{aligned}
W & \left\{x_{1}, \cdots, x_{m}, y_{1}, \cdots, y_{n}\right\} \\
= & \frac{1}{\left\{\pi^{m n}\left[x_{1}\left(x_{2}-x_{1}\right) \cdots\left(x_{m}-x_{m-1}\right)\right]^{n}\left[y_{1}\left(y_{2}-y_{1}\right) \cdots\left(y_{n}-y_{n-1}\right)\right]^{m}\right\}^{1 / 2}} \\
& \cdot \exp \left\{-\sum_{i=1}^{m} \sum_{j=1}^{n} \frac{\left[u_{i j}-u_{i, j-1}-u_{i-1, j}+u_{i-1, j-1}\right]^{2}}{\left(x_{i}-x_{i-1}\right)\left(y_{j}-y_{j-1}\right)}\right\}
\end{aligned}
$$

with the understanding that $u_{0, j}=u_{i, 0}=0$. Now in view of the fact that $m(I)$ for the interval $I$ as given by (2.1) is defined by means of the restriction points and the restricting set that appear in (2.1) and that the expression of an interval by restriction points and restricting set is not unique, it is necessary to prove the independence of the value $m(I)$ from the choice of restriction points and restricting set of $I$. This is done in $\S 3$.

3 . Uniqueness of the definition of the set function $m(I)$. To prove the uniqueness of the definition of $m(I)$ we show that when a finite number of partition points of the intervals $0 \leqq x \leqq 1,0 \leqq y \leqq 1$ are added and trivial re- 
strictions are added at the additional restriction points, the value of $m(I)$ remains unchanged. It suffices to consider, for instance, the introduction of an additional partition point $\xi$ satisfying $x_{k-1}<\xi<x_{k}$ together with trivial restrictions at $\left(\xi, y_{1}\right), \cdots,\left(\xi, y_{n}\right)$. With the partition points $x_{1}, \cdots, x_{k-1}, \xi$, $x_{k}, \cdots, x_{m}$ and $y_{1}, \cdots, y_{n}, m(I)$ is given according to (2.2), (2.3), by

$$
\begin{aligned}
m(I)= & \int_{E}^{(m n)} \int W\left\{x_{1}, \cdots, x_{m}, y_{1}, \cdots, y_{n}\right\} \\
& \cdot K\left\{x_{k-1}, \xi, x_{k}, y_{1}, \cdots, y_{n}\right\} d u_{11}, \cdots, d u_{m, n}
\end{aligned}
$$

where

$$
\begin{aligned}
K\left\{x_{k-1}, \xi, x_{k}, y_{1}, \cdots, y_{n}\right\} & \left(x_{k}-x_{k-1}\right)^{n / 2} \\
= & \frac{\pi^{n / 2}\left[\left(\xi-x_{k-1}\right)\left(x_{k}-\xi\right)\right]^{n / 2}\left[y_{1}\left(y_{2}-y_{1}\right) \cdots\left(y_{n}-y_{n-1}\right)\right]^{1 / 2}}{\left(x_{k}-\xi\right)\left(y_{j}-y_{j-1}\right)} \\
& \quad \int_{-\infty}^{\infty}(n) \int_{-\infty}^{\infty} \exp \left\{\sum _ { j = 1 } ^ { n } \left[-\frac{\left(u_{k, j}-u_{k, j-1}-v_{j}+v_{j-1}\right)^{2}}{\left(\xi-x_{k-1}\right)\left(y_{j}-y_{j-1}\right)}\right.\right. \\
& \left.\left.-\frac{\left(v_{j}-v_{j-1}-u_{k-1, j}+u_{k-1, j-1}\right)^{2}}{\left(x_{k}-x_{k-1}\right)\left(y_{j}-y_{j-1}\right)}\right]\right\} d v_{1} \cdots d v_{n} .
\end{aligned}
$$

Thus, for the uniqueness of $m(I)$ we only have to show that

$$
K\left\{x_{k-1}, \xi, x_{k}, y_{1}, \cdots, y_{n}\right\}=1 .
$$

In evaluating $K$ if we let $w_{1}=v_{1}, w_{2}=v_{2}-v_{1}, \cdots, w_{n}=v_{n}-v_{n-1}$, then the integral in the right hand of (3.2) becomes

$$
\begin{aligned}
\exp & \left\{\sum_{j=1}^{n} \frac{\left(u_{k, j}-u_{k, j-1}-u_{k-1, j}+u_{k-1, j-1}\right)^{2}}{\left(x_{k}-x_{k-1}\right)\left(y_{j}-y_{j-1}\right)}\right\} \\
\cdot \prod_{j=1}^{n}\left[\int _ { - \infty } ^ { \infty } \operatorname { e x p } \left\{-\frac{\left(u_{k, j}-u_{k, j-1}-w_{j}\right)^{2}}{\left(x_{k}-\xi\right)\left(y_{j}-y_{j-1}\right)}\right.\right. & \left.\left.-\frac{\left(w_{j}-u_{k-1, j}+u_{k-1, j-1}\right)^{2}}{\left(\xi-x_{k-1}\right)\left(y_{j}-y_{j-1}\right)}\right\} d w_{j}\right] .
\end{aligned}
$$

For the integrands in (3.3) we write

$$
\begin{aligned}
\frac{\left(u_{k, j}-u_{k, j-1}-w_{j}\right)^{2}}{\left(x_{k}-\xi\right)\left(y_{j}-y_{j-1}\right)} & +\frac{\left(w_{j}-u_{k-1, j}+u_{k-1, j-1}\right)^{2}}{\left(\xi-x_{k-1}\right)\left(y_{j}-y_{j-1}\right)} \\
& =A w_{j}^{2}+2 B w_{j}+C=A\left(w_{j}+\frac{B}{A}\right)^{2}+\left(C-\frac{B^{2}}{A}\right)
\end{aligned}
$$


where

$$
\begin{aligned}
A & =\frac{x_{k}-x_{k-1}}{\left(x_{k}-\xi\right)\left(\xi-x_{k-1}\right)\left(y_{j}-y_{j-1}\right)}, \\
B & =\frac{-u_{k-1, j}+u_{k-1, j-1}}{\left(\xi-x_{k-1}\right)\left(y_{j}-y_{j-1}\right)}-\frac{u_{k, j}-u_{k, j-1}}{\left(x_{k}-\xi\right)\left(y_{j}-y_{j-1}\right)},
\end{aligned}
$$

and

$$
C=\frac{\left(u_{k, j}-u_{k, j-1}\right)^{2}}{\left(x_{k}-\xi\right)\left(y_{j}-y_{j-1}\right)}+\frac{\left(-u_{k-1, j}+u_{k-1, j-1}\right)^{2}}{\left(\xi-x_{k-1}\right)\left(y_{j}-y_{j-1}\right)}
$$

and hence

$$
C-\frac{B^{2}}{A}=\frac{\left(u_{k, j}-u_{k, j-1}-u_{k-1, j}+u_{k-1, j-1}\right)^{2}}{\left(x_{k}-x_{k-1}\right)\left(y_{j}-y_{j-1}\right)} .
$$

With (3.4), (3.6), each of the $n$ integrals in (3.3) becomes

$$
\begin{aligned}
\exp \left\{-\frac{\left(u_{k, j}-u_{k, j-1}-u_{k-1, j}+u_{k-1, j-1}\right)^{2}}{\left(x_{k}-x_{k-1}\right)\left(y_{j}-y_{j-1}\right)}\right\} & \cdot \int_{-\infty}^{\infty} \exp \left\{-A\left(w_{j}+\frac{B}{A}\right)^{2}\right\} d w_{j}
\end{aligned}
$$

and finally with (3.5),

$$
\begin{aligned}
\int_{-\infty}^{\infty} \exp \left\{-A\left(w_{j}-\frac{B}{A}\right)^{2}\right\} d w_{j} & \\
= & \left(\frac{\pi}{A}\right)^{1 / 2}=\left[\frac{\pi\left(x_{k}-\xi\right)\left(\xi-x_{k-1}\right)\left(y_{j}-y_{j-1}\right)}{x_{k}-x_{k-1}}\right]^{1 / 2} .
\end{aligned}
$$

By (3.2), (3.3), (3.7), (3.8) it is seen readily that $K\left\{x_{k-1}, \xi, x_{k}, y_{1}, \cdots, y_{n}\right\}=1$ and the uniqueness of the definition of $m(I)$ is established.

4. Properties of $m(I)$. From (2.2), (2.3), the value of $m(I)$ is non-negative for any $I \in \mathcal{J}$. We point out here that $m(\varnothing)=0$ and $m\left(C_{2}\right)=1$. Let $0<x_{1} \leqq 1$, $0<y_{1} \leqq 1$, and $\varnothing$ and $C_{2}$ be given by

$$
\varnothing=\left\{f \in C_{2} ; f\left(x_{1}, y_{1}\right) \in \varnothing_{R_{1}}\right\} \quad \text { and } C_{2}\left\{f \in C_{2} ; f\left(x_{1}, y_{1}\right) \in R_{1}\right\}
$$

then by (2.2), (2.3)

$$
\begin{aligned}
& m(\varnothing)=\frac{1}{\left(\pi x_{1} y_{1}\right)^{1 / 2}} \int_{\phi_{R}} \exp \left\{-\frac{u_{11}^{2}}{x_{1} y_{1}}\right\} d u_{11}=0 \\
& m\left(C_{2}\right)=\frac{1}{\left(\pi x_{1} y_{1}\right)^{1 / 2}} \int_{R_{1}} \exp \left\{-\frac{u_{11}^{2}}{x_{1} y_{1}}\right\} d u_{11}=1
\end{aligned}
$$


We also point out that $m(I)$ is additive i.e. if $I_{1}, I_{2} \in \mathcal{J}, I_{1} \cap I_{2}=\varnothing$ and $I_{1} \cup I_{2} \in \mathcal{J}$ then $m\left(I_{1} \cup I_{2}\right)=m\left(I_{1}\right)+m\left(I_{2}\right)$. This can be easily seen if we extend, if necessary, the restricting sets of the two intervals to two sets in the same Euclidean space by adding restriction points and trivial restrictions on the additional restriction points.

5. Countable additivity of $m(I)$. To show that $m(I)$ is a measure on $g$ it remains to show that $m(I)$ is countably additive, i.e. if $\left\{I_{n}\right\}$ is a countable collection of elements of $\mathcal{g}$ such that $I_{i} \cap I_{j}=\varnothing$ for $i \neq j$ and $\bigcup_{n=1}^{\infty} I_{n} \in \mathcal{J}$, then $m\left(\cup_{n=1}^{\infty} I_{n}\right)=\sum_{n=1}^{\infty} m\left(I_{n}\right)$. In $\S 5$ we prove Theorem I which is an estimate of $m(I)$ when $I$ is an interval disjoint from a subset of $C_{2}$ defined by a Hölder condition. The proof of countable additivity of $m(I)$ is based on this estimate and is given in Theorem II, $\S 8$.

5.1. Theorem I. Let $\gamma$ be a fixed constant satisfying $0<\gamma<1 / 2$ and $a$ be an arbitrary positive number. Let a subset of $C_{2}, A_{a}$ be defined by

$$
\begin{aligned}
A_{a}=\left\{f \in C_{2} ; \mid\right. & f\left(x_{2}, y_{2}\right)-f\left(x_{1}, y_{1}\right) \mid \\
& \left.\leqq a\left[\left(x_{2}-x_{1}\right)^{2}+\left(y_{2}-y_{1}\right)^{2}\right]^{\gamma / 2}, 0 \leqq x_{1}, y_{1}, x_{2}, y_{2} \leqq 1\right\} .
\end{aligned}
$$

Then for any interval I disjoint from $A_{a}$

$$
m(I)<c a^{-8 /(1-2 \gamma)}
$$

where $c$ is a positive number and is independent of $a$.

We prove Theorem I by showing that if an element $f$ of $C_{2}$ does not belong to $A_{a}$ then it belongs to an element of a finite collection of intervals $\left\{J_{n}\right\}$ so that if $I \cap A_{a}=\varnothing$ then $I \subset \cup_{n} J_{n}$ and $m(I) \leqq \sum_{n} m\left(J_{n}\right)$. The intervals $J_{n}$ are so chosen that $\sum_{n} m\left(J_{n}\right)<c a^{-8 /(1-2 \gamma)}$. The above is done by means of Lemmas 1-4 which we prove in $\$ \S 5.2-5.5$. The proof of Theorem I itself is given in $\$ 5.6$.

\subsection{The first lemma.}

Notation. Let $n$ be a positive integer and consider two sequences of numbers $0=\xi_{0}<\xi_{1}<\cdots<\xi_{2^{n}}=1,0=\eta_{0}<\eta_{1}<\cdots<\eta_{2^{n}}=1$ where $\xi_{j}, \eta_{j}$ $=j / 2^{n}$ for $j=0,2,4,6, \cdots, 2^{n}$. Let $\beta^{k}$ be the collection of points of the unit square defined by

$$
\rho^{k}=\left\{P^{k}\{l, m\}=\left(\xi_{2^{k l}}, \eta_{2^{k m}}\right), l, m=0,1,2, \cdots, 2^{n-k}\right\}, \quad k=0,1,2, \cdots, n .
$$

We shall of ten write $P^{k}$ to mean a member of $\mathcal{P}^{k}$. Let $X^{k}\{l, m\}$ be the straight segment connecting the two points $P^{k}\{l-1, m\}$ and $P^{k}\{l, m\}$ for $l=1,2, \cdots$, $2^{n-k}, m=0,1,2, \cdots 2^{n-k}, k=0,1,2, \cdots, n$ and $Y^{k}\{l, m\}$ be the straight segment connecting the two points $P^{k}\{l, m-1\}$ and $P^{k}\{l, m\}$ for $l=0,1,2, \cdots, 2^{n-k}$, $m=1,2, \cdots, 2^{n-k}, k=0,1,2, \cdots, n$. When we are not particularly concerned about their positions we will write merely $X^{k}$ and $Y^{k}$ for $X^{k}\{l, m\}$ and $Y^{k}\{l, m\}$.

$\mathrm{By} \vartheta^{0}$ we mean the collection of right triangles with vertices $P^{0}\{l-1, m-1\}$, 
$P^{0}\{l, m-1\}, P^{0}\{l, m\}$ or with vertices $P^{0}\{l-1, m-1\}, P^{0}\{l-1, m\}$, $P^{0}\{l, m\},\left(l, m=1,2, \cdots, 2^{n}\right)$ and by $\mathfrak{Q}^{1}$ we mean the collection of squares with vertices $P^{1}\{l-1, m-1\}, P^{1}\{l, m-1\}, P^{1}\{l-1, m\}, P^{1}\{l, m\}$, $\left(l, m=1,2, \cdots, 2^{n-1}\right)$. The letter $Q$ is reserved for the unit square.

The length of $X^{k}\{l, m\}$ and $Y^{k}\{l, m\}$ will be denoted by $\lambda_{k}$. Then $\lambda_{k}$ $=2^{k} / 2^{n}=2^{k-n}$ independently of $l$ and $m$ in case $k=1,2, \cdots, n$, but when $k=0, \lambda_{k}$ depends on $l$ and $m$ and we only have the estimate $0<\lambda_{0}<\lambda_{1}=2^{1-n}$. Every element of $\mathfrak{Q}^{1}$ has four sides of equal length $\lambda_{1}$.

For any two points $P_{1}\left(x_{1}, y_{1}\right), P_{2}\left(x_{2}, y_{2}\right) \in Q$, the symbols $d\left(P_{1}, P_{2}\right)$, $d_{x}\left(P_{1}, P_{2}\right), d_{y}\left(P_{1}, P_{2}\right)$ mean

$$
\begin{gathered}
d\left(P_{1}, P_{2}\right)=\left[\left(x_{2}-x_{1}\right)^{2}+\left(y_{2}-y_{1}\right)^{2}\right]^{1 / 2}, \quad d_{x}\left(P_{1}, P_{2}\right)=\left|x_{2}-x_{1}\right|, \\
d y\left(P_{1}, P_{2}\right)=\left|y_{2}-y_{1}\right| .
\end{gathered}
$$

In connection with the above definitions we make a few remarks.

REMARK 1. From the definition of $\rho^{k}$ it follows that

$$
\rho^{k} \supset \rho^{k+1} \quad(k=0,1,2, \cdots, n-1) .
$$

Thus for any point on $Q$ which is also an element of $\rho^{0}$, the superscript $k$ is not unique. However the largest superscript for the point is unique and will be called the index of the point. Then it follows that if $k_{0}$ is the index of $P \in \mathcal{P}^{0}$, then $P \in \mathcal{P}^{k}$ for $k \leqq k_{0}$ and $P \notin \mathcal{P}^{k}$ for $k>k_{0}$. On the other hand for a segment on $Q$ which is an $X^{k}$ or a $Y^{k}$ the superscript $k$ is unique and such a segment will be referred to as an edge of index $k$.

REMARK 2. If $P=P^{k_{0}}\{l, m\}$ where $k_{0}$ is the index of $P$ and $P \in X^{k}$ then either $P$ is an end point of $X^{k}$ and hence $P \in \mathcal{P}^{k}, k_{0} \geqq k$, or $P$ is not an end

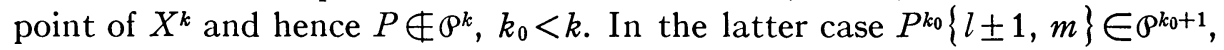
where $k_{0}+1 \leqq k$. A similar statement holds when $P \in Y^{k}$.

Remark 3. Suppose $P_{0}=P^{k_{0}}\left\{l, m_{0}\right\}$ where $k_{0}$ is the index of $P_{0}$ and $P_{0}$ is on some $Y^{k}$. If $P_{0}$ is not an end point of $Y^{k}$, then there is a sequence of points $P_{0}, P_{1}, P_{2}, \cdots, P_{\beta}$ on $Y^{k}$ with decreasing (or increasing, if you wish) $y$-coordinates such that the indices $k_{0}, k_{1}, k_{2}, \cdots, k_{\beta-1}$ of $P_{0}, P_{1}, P_{2}, \cdots$, $P_{\beta-1}$ satisfy $k_{0}<k_{1}<k_{2}<\cdots<k_{\beta}=k$; in particular, $P_{\beta}$ is an endpoint of $Y^{k}$ and $d\left(P_{0}, P_{1}\right)=\lambda_{k_{0}}, d\left(P_{1}, P_{2}\right)=\lambda_{k_{1}}, \cdots, d\left(P_{\beta-1}, P_{\beta}\right)=\lambda_{k_{\beta-1}}$. A similar statement holds when $P_{0}$ is on some $X^{k}$.

Proof. If $P_{0}$ is not an endpoint of $Y^{k}$, i.e. if $P_{0} \notin \rho^{k}$ then $k_{0}<k$. Let $P_{1}=P^{k_{0}}\left\{l, m_{0}-1\right\}$ which belongs to $\rho^{k_{0}+1}$ by Remark 2. If $P_{1} \in \rho^{k}$, let $P_{1}=P^{k}\left\{l_{1}, m_{1}\right\}$. If $P_{1} \notin \rho^{k}$, let $k_{1}$ be the index of $P_{1}$ and $P_{1}=P^{k_{1}}\left\{l_{1}, m_{1}\right\}$ where $k_{0}<k_{0}+1 \leqq k_{1}$ and $k_{1}<k$. We now repeat the process by letting $P_{2}$

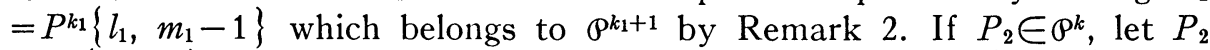
$=P^{k}\left\{l_{2}, m_{2}\right\}$. If $P_{2} \notin \odot^{k}$, let $k_{2}$ be the index of $P_{2}$ and $P_{2}=P^{k_{2}}\left\{l_{2}, m_{2}\right\}$ where $k_{1}<k_{1}+1 \leqq k_{2}$ and $k_{2}<k$. Thus repeating we obtain a strictly increasing sequence of integers $k_{0}<k_{1}<k_{2}<\cdots$ each of which satisfies $k_{i} \leqq k$. Since the difference between $k$ and $k_{0}$ is finite, $k$ is reached in a finite number of 
steps, say in $\beta$ steps. Then we have $P_{\beta} \in \mathcal{P}^{k_{\beta}}$ with $k_{\beta}=k$. By the choice of $P_{1}, P_{2}, \cdots, P_{\beta}$ it is clear that $d\left(P_{0}, P_{1}\right)=\lambda_{k_{0}}, d\left(P_{1}, P_{2}\right)=\lambda_{k_{1}}, \cdots, d\left(P_{\beta-1}, P_{\beta}\right)$ $=\lambda_{k_{\beta}}$.

We now state and prove Lemma 1 with the Remarks.

Lemma 1. Let $f(P)$ be a real valued function defined on $p^{\circ}$. Suppose $f(P)$ is such that if $P_{1}, P_{2}$ are the endpoints of some $X^{k}$ or $Y^{k}$ then

$$
\left|f\left(P_{2}\right)-f\left(P_{1}\right)\right| \leqq \operatorname{bad}^{\gamma}\left(P_{1}, P_{2}\right) \text { where } a>0, b=\left(1-2^{-\gamma}\right) / 16
$$

and

$$
0<\gamma<1 / 2
$$

Then for any $P_{1}, P_{2} \in \rho^{0}$,

$$
\left|f\left(P_{2}\right)-f\left(P_{1}\right)\right| \leqq a d^{\gamma}\left(P_{1}, P_{2}\right) .
$$

Proof. Let $P_{1}, P_{2} \in \mathcal{P}^{0}$ and $P_{1}=\left(\xi_{p}, \eta_{p^{\prime}}\right), P_{2}=\left(\xi_{q}, \eta_{q^{\prime}}\right)$ and $P_{3}=\left(\xi_{q}, \eta_{p^{\prime}}\right)$. Since $d^{\gamma}$ does not satisfy the triangle law, one cannot get a satisfactory estimate by going by short steps in a straight line from $P_{1}$ to $P_{3}$. In order to take fewer (and hence longer) steps, it is necessary to zigzag along the edges of larger and larger squares for which the inequality is known to hold. In this way the number of steps of each length is kept bounded, and the sum of $d^{r}$ distances can be satisfactorily estimated.

Consider $f\left(P_{3}\right)-f\left(P_{1}\right)$. If $p=q$, then $P_{1}=P_{3}$ and the difference is 0 . Therefore assume, without loss of generality in the proof, that $p<q$, and consider the set of integers $p, p+1, \cdots, q$. Everyone of these integers except possibly for $p$ which may be 0 , can be written as $l 2^{k}$ with an odd integer $l$. For an odd integer the exponent $k$ is 0 and for an even integer $k$ can not be 0 and is the largest possible integer for that numper because $l$ is an odd integer. Let $k$ be the greatest of all the unique exponents of $p, p+1, \cdots, q$. Let $r$ be a member of the set $\{p, p+1, \cdots, q\}$ with this greatest exponent $k$. Then $r$ is unique for the existence of two such numbers would contradict the maximality of $k$. Now let $r=l 2^{k}$. We have $p \leqq r \leqq q$ with $p<q$. Let $P_{3}^{*}=\left(\xi_{r}, \eta_{p^{\prime}}\right)$ and consider $f\left(P_{3}\right)-f\left(P_{3}^{*}\right)$. If $r=q$ then $P_{3}=P_{3}^{*}$ and the difference is 0 . Let us assume then that $r<q$ and express $q-r$ in the binary scale

$$
\begin{aligned}
& q-r=2^{k_{\alpha}}+2^{k_{\alpha-1}}+\cdots+2^{k_{1}}+2^{k_{0}} \\
& \text { with } k_{\alpha}>k_{\alpha-1}>\cdots>k_{1}>k_{0} \geqq 0 .
\end{aligned}
$$

We point out that $k>k_{\alpha}$, for otherwise the choice of $r$ would be contradicted.

It is clear that $P_{3}^{*}=\left(\xi_{r}, \eta_{p^{\prime}}\right)$ is on some $Y^{k}$ and $P_{3}=\left(\xi_{q}, \eta_{p^{\prime}}\right)$ is on some $Y^{k_{0}}$. If $P_{3}$ is not an endpoint of the $Y^{k_{0}}$ then according to Remark 3 there exists a sequence of points $P_{00}\left(=P_{3}\right), P_{01}, P_{02}, \cdots, P_{0, \beta_{0}}$ on the $Y^{k_{0}}$ with decreasing $y$ coordinates such that the indices $k_{00}, k_{01}, k_{02}, \cdots, k_{0, \beta_{0}-1}$ of $P_{00}, P_{01}, P_{02}$, $\cdots, P_{0, \beta_{0}-1}$ satisfy $k_{00}<k_{01}<k_{02}<\cdots<k_{0, \beta_{0}}=k_{0}$ and furthermore 
$d\left(P_{00}, P_{01}\right)=\lambda_{k_{00}}, d\left(P_{01}, P_{02}\right)=\lambda_{k_{01}}, \cdots, d\left(P_{0, \beta_{0}-1}, P_{0, \beta_{0}}\right)=\lambda_{k_{0}, \beta_{0}-1}$, and in particular $P_{0, \beta_{0}}$ is an endpoint of the $Y^{k_{0}}$. Let $P_{0, \beta_{0}}=P^{k_{0}}\left\{l_{0}, m_{0}\right\}$. If $P_{3}$ is an endpoint of the $Y^{k_{0}}$ to begin with, let $P_{3}=P_{0, \beta_{0}}=P^{k_{0}}\left\{l_{0}, m_{0}\right\}$ with $\beta_{0}=0$. In any case $P^{k_{0}}\left\{l_{0}-1, m_{0}\right\}$ is on some $Y^{k_{1}}$ by (5.5). Now we iterate the above process. If $P^{k_{0}}\left\{l_{0}-1, m_{0}\right\}$ is not an endpoint of the $Y^{k_{1}}$, then according to Remark 3, there exists a sequence of points $P_{10}\left(=P^{k_{0}}\left\{l_{0}-1, m_{0}\right\}\right), P_{11}, P_{12}, \cdots, P_{1, \beta_{1}}$ on the $Y^{k_{1}}$ with decreasing $y$ coordinates such that the indices $k_{10}, k_{11}, k_{12}$, $\cdots, k_{1, \beta_{1}-1}$ of $P_{10}, P_{11}, P_{12}, \cdots, P_{1, \beta_{1}-1}$ satisfy $k_{0} \leqq k_{10}<k_{11}<k_{12}<\cdots$ $<k_{1, \beta_{1}}=k_{1}, d\left(P_{10}, P_{11}\right)=\lambda_{k_{10}}, d\left(P_{11}, P_{12}\right)=\lambda_{k_{11}}, \cdots, d\left(P_{1, \beta_{1}-1}, P_{1, \beta_{1}}\right)=\lambda_{k_{1}, \beta_{1}-1}$ and in particular $P_{1, \beta_{1}}$ is an endpoint of the $Y^{k_{1}}$. Let $P_{1, \beta_{1}}=P^{k_{1}}\left\{l_{1}, m_{1}\right\}$. If $P^{k_{0}}\left\{l_{0}-1, m_{0}\right\}$ is an endpoint of $Y^{k_{1}}$ to begin with, let $P^{k_{0}}\left(l_{0}-1, m_{0}\right)=P_{1, \beta_{1}}$ $=P^{k_{1}}\left(l_{1}, m_{1}\right)$ with $\beta_{1}=0$. In any case $P^{k_{1}}\left(l_{1}-1, m_{1}\right)$ is on some $Y^{k_{2}}$ by (5.5). Thus repeating we reach a point $P_{\alpha, \beta_{\alpha}}=P^{k}\left\{l_{\alpha}, m_{\alpha}\right\}$ which is an endpoint of some $Y^{k_{\alpha}}$. Then by (5.5), $P^{k_{\alpha}}\left\{l_{\alpha}-1, m_{\alpha}\right\}$ is on some $Y^{k}$ and has the same $x$ coordinate as $P_{3}^{*}$. Let $P^{k_{\alpha}}\left\{l_{\alpha}-1, m_{\alpha}\right\}=P_{\alpha+1,0}=P^{k_{\alpha+1}}\left\{l_{\alpha+1}, m_{\alpha+1}\right\}$ with $k_{\alpha, \beta_{\alpha}}$ $\leqq k_{\alpha+1} \leqq k$. In this manner we construct a sequence of points which consists of $\alpha+2$ subsequences, $\left\{P_{00}, P_{01}, P_{02}, \cdots, P_{0, \beta_{0}}\right\},\left\{P_{10}, P_{11}, P_{12}, \cdots, P_{1, \beta_{1}}\right\}$, $\cdots,\left\{P_{\alpha, 0}, P_{\alpha, 1}, P_{\alpha, 2}, \cdots, P_{\alpha, \beta_{\alpha}}\right\},\left\{P_{\alpha+1,0}\right\}$ with $\beta_{0}, \beta_{1}, \cdots, \beta_{\alpha} \geqq 0$ and a sequence of integers which consists of $\alpha+2$ subsequences

$$
\begin{aligned}
\left\{k_{00}, k_{01}, k_{02}, \cdots, k_{0, \beta_{0}}\right\},\left\{k_{10}, k_{11}, k_{12}, \cdots,\right. & \left.k_{1, \beta_{1}}\right\}, \cdots, \\
& \cdot\left\{k_{\alpha, 0}, k_{\alpha, 1}, k_{\alpha, 2}, \cdots, k_{\alpha, \beta_{\alpha}}\right\},\left\{k_{\alpha+1}\right\}
\end{aligned}
$$

with the property $k_{00}<k_{11}<k_{12}<\cdots<k_{0, \beta_{0}} \leqq k_{10}<k_{11}<k_{12}<\cdots<k_{1, \beta_{1}}$ $\leqq k_{20}<\cdots<k_{\alpha-1, \beta_{\alpha-1}} \leqq k_{\alpha, 0}<k_{\alpha, 1}<\alpha_{2,2}<\cdots<k_{\alpha, \beta_{\alpha}} \leqq k_{\alpha+1}$. Furthermore $P_{0, \beta_{0}}, P_{1, \beta_{1}}, \cdots, P_{\alpha, \beta_{\alpha}}, P_{\alpha+1,0}$ have $k_{0, \beta_{0}}=k_{0}, k_{1, \beta_{1}}=k_{1}, \cdots, k_{\alpha, \beta_{\alpha}}=k_{\alpha}, k_{\alpha+1}$ as their superscripts and each $P_{i, j}$ of the rest of the sequence has $k_{i, j}$ as its index. Any two successive points in the sequence are the endpoints of an edge whose index is equal to the suffix of the first of the two points. This edge is parallel to the $y$-axis in case the two points belong to one of the $\alpha+2$ subsequences and it is parallel to the $x$-axis if they belong to two consecutive subsequences. In particular $P_{00}=P_{3}$ and $P_{\alpha+1,0}$ has the same $x$-coordinate as $P_{3}^{*}$.

Let the projection on the $Y^{k}$ containing $P_{3}^{*}$ of each member $P_{i, j}$ of the sequence $P_{00}, P_{01}, \cdots, P_{\alpha+1,0}$ be denoted by $P_{i, j}^{*}$ and consider the sequence $P_{00}^{*}, P_{10}^{*}, \cdots, P_{\alpha+1,0}^{*}$. Any two successive points are the endpoints of an edge parallel to the $y$-axis with an index which is equal to the suffix of the first of the two points provided the preimages of these two points belong to one of the $\alpha+2$ subsequences, otherwise they are coincident. We notice that in particular $P_{\alpha+1,0}^{*}=P_{\alpha+1,0}$. Let us use the sequence of points $P_{00}\left(=P_{3}\right), P_{0,1}$, $\cdots, P_{\alpha, \beta_{\alpha}}, P_{\alpha+1,0}\left(=P_{\alpha+1,0}^{*}=P_{\alpha, \alpha_{\beta}}^{*}\right), P_{\alpha, \alpha_{\beta}-1}^{*}, \cdots, P_{01}^{*}, P_{00}^{*}\left(=P_{3}^{*}\right)$ as intermediate points between $P_{3}$ and $P_{3}^{*}$ in estimating $f\left(P_{3}\right)-f\left(P_{3}^{*}\right)$. Then by (5.3)

$$
\left|f\left(P_{3}\right)-f\left(P_{3}^{*}\right)\right| \leqq 2 b a \sum_{i=0}^{k_{\alpha}} \lambda_{i}^{\gamma}<2 b a\left(2^{(1-n) \gamma}+\sum_{i=1}^{k_{\alpha}} 2^{(i-n) \gamma}\right) .
$$


From the fact that $0<\gamma<1 / 2$, it follows that $2^{\gamma}<1 /\left(1-2^{-\gamma}\right), 2^{(1-n) \gamma}$ $<2^{-n \gamma} /\left(1-2^{-\gamma}\right)=\sum_{i=0}^{-\infty} 2^{(i-n) \gamma}$ and hence

$$
\begin{aligned}
\left|f\left(P_{3}\right)-f\left(P_{3}^{*}\right)\right| & \leqq 2 b a \sum_{i=k_{\alpha}}^{-\infty} 2^{(i-n) \gamma} \\
& =2 b a 2^{\left(k_{\alpha}-n\right) \gamma} \sum_{i=k_{\alpha}}^{-\infty} 2^{\left(i-k_{\alpha}\right) \gamma}=\frac{2 b a}{1-2^{-\gamma}} 2^{\left(k_{\alpha}-n\right) \gamma} .
\end{aligned}
$$

Let us then estimate $d\left(P_{3}, P_{3}^{*}\right)$. The $x$-coordinates of $P_{3}$ and $P_{3}^{*}$ are $\xi_{q}$ and $\xi_{r}$ and $q-r$ is given by (5.5). If $k_{\alpha}=0$ or 1 , in (5.5), then $q-r \leqq 3$ and by (5.3)

$$
\left|f\left(P_{3}\right)-f\left(P_{3}^{*}\right)\right|<3 b a d^{\gamma}\left(P_{3}, P_{3}^{*}\right)<\frac{3 b a}{1-2^{-\gamma}} d^{\gamma}\left(P_{3}, P_{3}^{*}\right)
$$

$$
\text { for } k_{\alpha}=0,1 \text {. }
$$

Let $k_{\alpha} \geqq 2$. Since $\xi_{q}>(q-1) / 2^{n}$ whether $q$ is even or odd and similarly $\xi_{r}<(r+1) / 2^{n}$ we have, according to $(5.5)$

$$
d\left(P_{3}, P_{3}^{*}\right)=\xi_{q}-\xi_{r} \geqq \frac{(q-1)-(r+1)}{2^{n}} \geqq \frac{2^{k_{\alpha}}-2}{2^{n}} \geqq \frac{1}{2} 2^{k_{\alpha}-n},
$$

$$
d^{\gamma}\left(P_{3}, P_{3}^{*}\right) \geqq \frac{1}{2} 2^{\left(k_{\alpha}-n\right) \gamma} .
$$

From (5.6), (5.8)

$$
\left|f\left(P_{3}\right)-f\left(P_{3}^{*}\right)\right|<\frac{4 b a}{1-2^{-\gamma}} d^{\gamma}\left(P_{3}, P_{3}^{*}\right) \quad \text { for } \quad k_{\alpha} \geqq 2 .
$$

By (5.7), (5.9)

$$
\left|f\left(P_{3}\right)-f\left(P_{3}^{*}\right)\right|<\frac{4 b a}{1-2^{-\gamma}} d^{\gamma}\left(P_{3}, P_{3}^{*}\right) .
$$

Exactly the same estimate as (5.10) holds for the pair $P_{1}$ and $P_{3}^{*}$ and from the fact $d\left(P_{1}, P_{3}\right) \geqq d\left(P_{3}, P_{3}^{*}\right), d\left(P_{1}, P_{3}^{*}\right)$, we have

$$
\left|f\left(P_{3}\right)-f\left(P_{1}\right)\right|<\frac{8 b a}{1-2^{-\gamma}} d^{\gamma}\left(P_{1}, P_{3}\right) .
$$

Again exactly the same estimate as (5.11) holds for the pair $P_{2}$ and $P_{3}$ and from the fact that $d\left(P_{1}, P_{2}\right) \geqq d\left(P_{1}, P_{3}\right), d\left(P_{2}, P_{3}\right)$ and

$$
\left|f\left(P_{2}\right)-f\left(P_{1}\right)\right|<\frac{16 b a}{1-2^{-\gamma}} d^{\gamma}\left(P_{1}, P_{2}\right)=a d^{\gamma}\left(P_{1}, P_{2}\right) .
$$

This completes the proof of Lemma 1. 
5.3. Lemma 2. Let a real number be assigned to each element of $\mathcal{P}^{0}$. Let $f(P)$ be defined on the unit square $Q$ in such a way that $f(P)$ takes on the preassigned values on $\boldsymbol{P}^{0}$, is linear on each element of the collection of triangles $\vartheta^{0}$ and for any $P_{1}, P_{2} \in \otimes^{0}$

$$
\left|f\left(P_{2}\right)-f\left(P_{1}\right)\right| \leqq a d^{\gamma}\left(P_{1}, P_{2}\right) .
$$

Then for any $P_{1}, P_{2} \in Q$,

$$
\left|f\left(P_{2}\right)-f\left(P_{1}\right)\right| \leqq 22 a d^{r}\left(P_{1}, P_{2}\right) .
$$

Proof. Let $P_{1}, P_{2} \in Q$ be arbitrarily given. Let $P_{3}$ have the same $x$ coordinate as $P_{2}$ and the same $y$-coordinate as $P_{1}$. Consider $f\left(P_{3}\right)-f\left(P_{1}\right)$.

CASE 1. If $P_{1}, P_{3}$ are contained in one element of $\vartheta^{0}$ let $P_{1}^{*}, P_{3}^{*}$ be the projections of $P_{1}, P_{3}$ on the side $P_{4} P_{5}$ of the triangle which is parallel to the $x$-axis. Then from the linearity of $f(P)$ on the triangle and by (5.12)

$$
\begin{aligned}
\left|f\left(P_{3}\right)-f\left(P_{1}\right)\right| & =\left|f\left(P_{3}^{*}\right)-f\left(P_{1}^{*}\right)\right|=\frac{d\left(P_{1}, P_{3}\right)}{d\left(P_{4}, P_{5}\right)}\left|f\left(P_{5}^{*}\right)-f\left(P_{4}^{*}\right)\right| \\
& \leqq \frac{d^{\gamma}\left(P_{1}, P_{3}\right)}{d^{\gamma}\left(P_{4}, P_{5}\right)}\left|f\left(P_{5}^{*}\right)-f\left(P_{4}^{*}\right)\right| \leqq a d^{\gamma}\left(P_{1}, P_{3}\right) .
\end{aligned}
$$

CASE 2. If $P_{1}, P_{3}$ are contained in one element of $\mathfrak{Q}^{1}$, then the segment $P_{1} P_{3}$ is contained in at most four elements of $\vartheta^{0}$. Using the intersections of $P_{1} P_{3}$ with the boundaries of these triangles as intermediate points between $P_{1}$ and $P_{3}$ we have by $(5.14)$

$$
\left|f\left(P_{3}\right)-f\left(P_{1}\right)\right| \leqq 4 a d^{r}\left(P_{1}, P_{3}\right) .
$$

CASE 3. If $P_{1}, P_{3}$ are contained in two adjacent elements of $\mathfrak{Q}^{1}$ then the segment $P_{1} P_{3}$ is contained in at most eight elements of $\vartheta^{0}$ and hence

$$
\left|f\left(P_{3}\right)-f\left(P_{1}\right)\right| \leqq 8 a d^{\gamma}\left(P_{1}, P_{3}\right) .
$$

CASE 4. Finally when $P_{1}, P_{3}$ are not in one or two adjacent elements of $\mathfrak{Q}^{1}$, let $Q_{1}^{1}, Q_{3}^{1}$ be the two elements of $\mathfrak{Q}^{1}$ which contain $P_{1}, P_{3}$ respectively. Let the intersections of the segment $P_{1} P_{3}$ with the boundaries of $Q_{1}^{1}, Q_{3}^{1}$ be $P_{11}, P_{31}$ respectively. Of the two endpoints of the edge of $Q_{1}^{1}$ that contains $P_{11}$, let $P_{12}$ be the one contained in the same element of $\vartheta^{0}$ as $P_{11}$ and let $P_{32}$ be chosen in the same way. Then $P_{1}, P_{32}$ have the same $y$-coordinates. Consider the chain of points $P_{1}, P_{11}, P_{12}, P_{32}, P_{31}, P_{3}$. For the pair $P_{1}, P_{11}$ and the pair $P_{31}, P_{3}$, the estimate (5.15) holds; for the pair $P_{11}, P_{12}$ and the pair $P_{32}$, $P_{31}$, the estimate (5.14) holds and for the pair $P_{12}, P_{32}$ the assumption (5.12) holds. Furthermore $d\left(P_{1}, P_{3}\right) \geqq d\left(P_{12}, P_{32}\right)$ and $d\left(P_{12}, P_{32}\right)$ is greater than any one of $d\left(P_{1}, P_{11}\right), d\left(P_{11}, P_{12}\right), d\left(P_{32}, P_{31}\right), d\left(P_{31}, P_{3}\right)$. Hence

$$
\left|f\left(P_{3}\right)-f\left(P_{1}\right)\right|<11 a d^{\gamma}\left(P_{1}, P_{3}\right) .
$$


From (5.14), (5.15), (5.16), (5.17), the estimate (5.17) for $P_{1}, P_{3}$ for all cases holds and with exactly the same estimate for the pair $P_{1}, P_{2}$ we conclude that

$$
\left|f\left(P_{2}\right)-f\left(P_{1}\right)\right|<22 a d^{\gamma}\left(P_{1}, P_{2}\right) .
$$

Combining Lemma 1 and Lemma 2 we have:

5.4. Lemma 3. Let $f(P)$ be a real valued function defined and continuous on the unit square $Q$ and linear on each element of $\vartheta^{0}$. Furthermore let $f(P)$ be such that if $P_{1}, P_{2}$ are the end points of some $X^{k}$ or $Y^{k}$

$$
\left|f\left(P_{2}\right)-f\left(P_{1}\right)\right| \leqq \frac{1}{22 \times 16}\left(1-2^{-\gamma}\right) a d^{\gamma}\left(P_{1}, P_{2}\right)
$$

holds. Then for any $P_{1}, P_{2} \in Q$

$$
\left|f\left(P_{2}\right)-f\left(P_{1}\right)\right| \leqq a d^{\gamma}\left(P_{1}, P_{2}\right) .
$$

5.5. Lemma 4. Let $0 \leqq x_{1}<x_{2} \leqq 1,0 \leqq y_{1}<y_{2} \leqq 1$. Then for the interval

$$
I=\left\{f \in C_{2} ;\left|f\left(x_{2}, y_{1}\right)-f\left(x_{1}, y_{1}\right)\right|>a\left|x_{2}-x_{1}\right|^{\gamma}\right\},
$$

$m(I)=0$ in case $y_{1}=0$ and if $y_{1} \neq 0$

$$
m(I) \leqq \exp \left\{-\frac{a^{2}}{\left(x_{2}-x_{1}\right)^{1-2 \gamma} y_{1}}\right\} .
$$

Similar estimates hold for the interval

$$
I=\left\{f \in C_{2} ;\left|f\left(x_{1}, y_{2}\right)-f\left(x_{1}, y_{1}\right)\right|>a\left|y_{2}-y_{1}\right| \gamma\right\} .
$$

Proof. The set $I$ given by (5.20) is an interval with restriction points $\left(x_{1}, y_{1}\right),\left(x_{2}, y_{1}\right)$ and restricting set $E=\left\{\left(u_{11}, u_{21}\right) \in R_{2} ;\left|u_{21}-u_{11}\right|>a\left|x_{2}-x_{1}\right| \gamma\right\}$, a Lebesgue measurable set in $R_{2}$. In case $y_{1}=0$, then since $f(x, 0)=0$ for $0 \leqq x \leqq 1$, the interval defined by (5.20) is an empty set and $m(I)=0$.

Let $y_{1} \neq 0$. By (2.2), (2.3)

$$
m(I)=\frac{1}{\pi\left[x_{1}\left(x_{2}-x_{1}\right)\right]^{1 / 2} y_{1}} \iint_{E} \exp \left\{-\frac{u_{11}^{2}}{x_{1} y_{1}}-\frac{\left(u_{21}-u_{11}\right)^{2}}{\left(x_{2}-x_{1}\right) y_{1}}\right\} d u_{11} d u_{12} .
$$

With the transformation $v_{1}=u_{11}, v_{2}=u_{21}-u_{11}$, the restricting set $E$ becomes $E=\left\{\left(v_{1}, v_{2}\right) \in R_{2},-\infty<v_{1}<\infty,\left|v_{2}\right|>b\right\}$ where $b=a\left|x_{2}-x_{1}\right|^{\gamma}$. Thus

$$
\begin{aligned}
m(I)= & \frac{1}{\pi\left[x_{1}\left(x_{2}-x_{1}\right)\right]^{1 / 2} y_{1}}\left\{\int_{-\infty}^{\infty} \exp \left\{-\frac{v_{1}^{2}}{x_{1} y_{1}}\right\} d v_{1}\right\} \\
& \cdot\left\{\int_{\left|v_{2}\right|>b} \exp \left\{-\frac{v_{2}^{2}}{\left(x_{2}-x_{1}\right) y_{1}}\right\} d v_{2}\right\} .
\end{aligned}
$$


The value of the first integral is $\left(\pi x_{1} y_{1}\right)^{1 / 2}$. To estimate the second integral let $w=v_{2}-b ;$ then

$$
\begin{aligned}
\int_{\left|\boldsymbol{v}_{2}\right|>b} \exp \left\{\frac{-v_{2}^{2}}{\left(x_{2}-x_{1}\right) y_{1}}\right\} d v_{2} & =2 \int_{0}^{\infty} \exp \left\{\frac{-(w+b)^{2}}{\left(x_{2}-x_{1}\right) y_{1}}\right\} d w \\
& \leqq 2 \exp \left\{\frac{-b^{2}}{\left(x_{2}-x_{1}\right) y_{1}}\right\} \int_{0}^{\infty} \exp \left\{\frac{-w^{2}}{\left(x_{2}-x_{1}\right) y_{1}}\right\} d w
\end{aligned}
$$

The value of the last integral is $\left(\pi\left(x_{2}-x_{1}\right) y_{1}\right)^{1 / 2} / 2$. From these evaluations and estimates, (5.21) follows.

5.5. Proof of Theorem I. With notations introduced in $\$ 5.2$, the set $A_{a}$ may be written: $A_{a}=\left\{f \in C_{2} ;\left|f\left(P_{2}\right)-f\left(P_{1}\right)\right| \leqq \operatorname{ad}^{\gamma}\left(P_{1}, P_{2}\right), P_{1}, P_{2} \in Q\right\}$. Let $I \in \mathcal{J}, \quad I \cap A_{a}=\varnothing$ and $I=\left\{f \in C_{2}, \quad\left[f\left(x_{1}, y_{1}\right), \cdots, f\left(x_{s}, y_{t}\right)\right] \in E\right\}$ where $0<x_{1}<x_{2}<\cdots<x_{s} \leqq 1,0<y_{1}<y_{2}<\cdots<y_{t} \leqq 1$ and $E$ is a Lebesgue measurable set in $R_{s t}$. Let $n$ be so large that

$$
2^{1-n}<\min \left\{x_{1}, x_{2}-x_{1}, \cdots, x_{s}-x_{s-1}, y_{1}, y_{2}-y_{1}, \cdots, y_{t}-y_{t-1}\right\}
$$

and $2^{1-n}<1-x_{s}, 1-y_{t}$ in case $x_{s} \neq 1, y_{t} \neq 1$. Consider partitions of the intervals $0 \leqq x \leqq 1,0 \leqq y \leqq 1$ into the subintervals of length $2^{1-n}$ by the partition points $\xi_{2 l}=2 l / 2^{n}\left(l=0,1,2, \cdots, 2^{n-1}\right), \eta_{2 m}=2 m / 2^{n},\left(m=0,1,2, \cdots, 2^{n-1}\right)$. Each interval $\xi_{2(l-1)}<x<\xi_{2 l}$ contains at most one $x_{i},(i=1,2, \cdots, s)$. If it contains one $x_{i}$ let $\xi_{2 l-1}=x_{i}$; otherwise let $\xi_{2 l-1}=\left(\xi_{2(l-1)}+\xi_{2 l}\right) / 2$. Then $\left\{x_{1}, x_{2}, \cdots, x_{s}\right\} \subset\left\{\xi_{1}, \xi_{2}, \cdots, \xi_{2^{n}}\right\}$. We choose odd numbered $\eta_{j}$ in the same fashion so that $\left\{y_{1}, y_{2}, \cdots, y_{t}\right\} \subset\left\{\eta_{1}, \eta_{2}, \cdots, \eta_{2^{n}}\right\}$. Let

$$
\rho^{0}=\left\{\left(\xi_{1}, \eta_{1}\right), \cdots,\left(\xi_{2^{n}}, \eta_{2^{n}}\right)\right\} \supset\left\{\left(x_{1}, y_{1}\right), \cdots,\left(x_{s}, y_{t}\right)\right\} .
$$

Let $\rho^{0}$ be restriction points of $I$ by adding trivial restrictions at the additional restriction points and extending $E$ to a Lebesgue measurable set $E^{\prime}$ in $R_{4}{ }^{n}$.

For any $f \in I$, let $f^{*}$ be such that $f^{*}=f$ on $\beta^{0}$ and $f^{*}$ is linear on each element of $\vartheta^{0}$. Since $f^{*}$ agrees with $f$ on the collection of restriction points $\beta^{0}$ of $I, f^{*} \in I$ and hence $f^{*} \notin A_{a}$. Then by Lemma $3 f^{*}$ must violate (5.18) at the endpoints of some $X^{k}$ or $Y^{k}$. Since $f$ agrees with $f^{*}$ on $\rho^{0}$ which contains endpoints of $X^{k}, Y^{k} f$ violates (5.18) at the endpoints of some $X^{k}$ or $Y^{k}$. In other words $f \in J_{x}\{k, l, m\}$ or $f \in J_{y}\{k, l, m\}$ for some $k=0,1,2, \cdots, n ; l, m$ $=1,2, \cdots, 2^{n-k}$ where

$$
\begin{aligned}
J_{x}\{k, l, m\} & =\left\{f \in C_{2} ;\left|f\left(P^{k}\{l, m\}\right)-f\left(P^{k}\{l-1, m\}\right)\right|\right. \\
& >b d^{\gamma}\left(P^{k}\{l-1, m\}, P^{k}\{l, m\}\right), \\
J_{y}\{k, l, m\} & =\left\{f \in C_{2} ;\left|f\left(P^{k}\{l, m\}\right)-f\left(P^{k}\{l, m-1\}\right)\right|\right. \\
& >b d^{\gamma}\left(P^{k}\{l, m-1\}, P^{k}\{l, m\}\right),
\end{aligned}
$$




$$
b=\frac{1}{22 \times 16}\left(1-2^{-\gamma}\right) a .
$$

We remember that $J_{x}\{k, l, 0\}=J_{y}\{k, 0, m\}=\varnothing$.

It follows then that

$$
I \subset \bigcup_{k=0}^{n} \bigcup_{l=1}^{2^{n-k}} \bigcup_{m=1}^{2^{n-k}}\left(J_{x}\{k, l, m\} \cup J_{y}\{k, l, m\}\right),
$$

and from the finite additivity of $m$

$$
m(I) \leqq \sum_{k=0}^{n} \sum_{l=1}^{2^{n-k}} \sum_{m=1}^{2^{n-k}}\left\{m\left(J_{x}\{k, l, m\}\right)+m\left(J_{y}\{k, l, m\}\right)\right\} .
$$

By Lemma 4

$$
m\left(J_{x}\{k, l, m\}\right) \leqq \exp \left\{\frac{-b^{2}}{\left(\xi_{l 2^{k}}-\xi_{(l-1) 2^{k}}\right)^{1-2 \gamma_{\eta_{2} 2^{k}}}}\right\} \leqq \exp \left\{\frac{-b^{2}}{\left(\xi_{l 2^{k}}-\xi_{(l-1) 2^{k}}\right)^{1-2 \gamma}}\right\},
$$

and from the independence of the last estimate from $m$ and from the independence and the similar estimate for $m\left(J_{y}\{k, l, m\}\right)$ from $l$

$$
m(I) \leqq 2 \sum_{k=0}^{n} \sum_{l=1}^{2^{n-k}} 2^{n-k} \exp \left\{\frac{-b^{2}}{\left(\xi_{l 2^{k}}-\xi_{(l-1) 2^{k}}\right)^{1-2 \gamma}}\right\} .
$$

In the summation above, for $k=0, \xi_{l}-\xi_{l-1}<2 / 2^{n}$ and for $k \geqq 1, \xi_{l 2^{k}}-\xi_{(l-1) 2^{k}}$ $=2^{k-n}$ and consequently

$$
m(I) \leqq 2\left[4^{n} \exp \left\{-b^{2} 2^{(n-1)(1-2 \gamma)}\right\}+\sum_{k=1}^{n} 4^{n-k} \exp \left\{-b^{2} 2^{(n-k)(1-2 \gamma)}\right\}\right] .
$$

Reversing the order of summation in the second term above and then noticing that the first term above is four times the $(n-1)$ st member of the second term after the reversion, we have

$$
\begin{aligned}
m(I) & \leqq 2\left[4 \times 4^{n-1} \exp \left\{-b^{2} 2^{(n-1)(1-2 \gamma)}\right\}+\sum_{k=1}^{n} 4^{k-1} \exp \left\{-b^{2} 2^{(k-1)(1-2 \gamma)}\right\}\right] \\
& <10 \sum_{k=1}^{\infty} 4^{k-1} \exp \left\{-b^{2} 2^{(k-1)(1-2 \gamma)}\right\} \\
& =10 \sum_{k=0}^{\infty} 4^{-k} 4^{2 k} \exp \left\{-b^{2} 2^{2 k(1-2 \gamma) / 2}\right\} .
\end{aligned}
$$

To estimate the last series let us consider the function $\phi(u)=u^{2} \exp \left\{-b^{2} u^{\lambda}\right\}$ for $u \geqq 0$ with $\lambda>0$. This function has exactly one maximum at $u_{\max }=\left(2 / \lambda b^{2}\right)^{1 / \lambda}$ and the maximum value is given by $\phi\left(u_{\max }\right)=\left(\lambda b^{2} e / 2\right)^{-2 / \lambda}$. If we identify $u$ with $2^{2 k}$ and $\lambda$ with $(1-2 \gamma) / 2$ in the last member of $(5.23)$ then 


$$
4^{2 k} \exp \left\{-b^{2} 2^{2 k(1-2 \gamma) / 2}\right\} \leqq\left\{\frac{(1-2 \gamma) b^{2} e}{4}\right\}^{-4 /(1-2 \gamma)},
$$

and hence from (5.23), (5.22),

$$
\begin{aligned}
m(I) & \leqq 10\left\{\frac{(1-2 \gamma) b^{2} e}{4}\right\}^{-4 /(1-2 \gamma)} \sum_{k=0}^{\infty} 4^{-k} \\
& =\frac{40}{3}\left\{\frac{(1-2 \gamma)\left(1-2^{-\gamma}\right)^{2} e}{(2 \times 16 \times 22)^{2}}\right\}^{-4 /(1-2 \gamma)} a^{-8 /(1-2 \gamma)} .
\end{aligned}
$$

This completes the proof of Theorem I.

6. Compactness of $A_{a}$.

Lemma 5. The subset $A_{a}$ of $C_{2}$ defined by

$$
A_{a}=\left\{f \in C_{2}:\left|f\left(P_{2}\right)-f\left(P_{1}\right)\right| \leqq a d^{\gamma}\left(P_{1}, P_{2}\right), P_{1}, P_{2} \in Q\right\}
$$

is compact in itself in the weak topology of $C_{2}$, i.e. for any sequence $\left\{f_{n}\right\} \subset A_{a}$ there exists a subsequence $\left\{f_{n_{k}}\right\}$ which converges point-wise on $Q$ to an element $f_{0} \in A_{a}$.

Proof. Let $0=(0,0)$. Then for any $f \in A_{a}, P \in Q$,

$$
|f(P)|=|f(P)-f(0)| \leqq a d^{\gamma}(P, 0) \leqq 2^{1 / 2} a .
$$

Let $\left\{f_{n}\right\}$ be an arbitrary sequence of elements of $A_{a}$ and $\left\{P_{\alpha}\right\}$ be a countable collection of points which is dense in $Q$ in the usual topology. For each $P_{\alpha}$, the sequence of numbers $\left\{f_{n}\left(P_{\alpha}\right)\right\}$ is bounded according to (6.1) and hence has a convergent subsequence. Let $\left\{f_{1, n}\right\}$ be a subsequence of $\left\{f_{n}\right\}$ which converges at $P_{1},\left\{f_{2, n}\right\}$ be a subsequence of $\left\{f_{1, n}\right\}$ which converges at $P_{2}$ and so forth. Then the subsequence $\left\{g_{n}\right\}$ of $\left\{f_{n}\right\}$ where $g_{n}=f_{n, n}$ converges on $\left\{P_{\alpha}\right\}$. Let us define a function $f_{0}$ on $\left\{P_{\alpha}\right\}$ by $f_{0}\left(P_{\alpha}\right)=\lim _{n \rightarrow \infty} g_{n}\left(P_{\alpha}\right)$. For any $P_{\alpha_{1}}, P_{\alpha_{2}} \in\left\{P_{\alpha}\right\}$, since $g_{n} \in A_{a}$,

$$
\left|f_{0}\left(P_{\alpha_{2}}\right)-f_{0}\left(P_{\alpha_{1}}\right)\right|=\lim _{n \rightarrow \infty}\left|g_{n}\left(P_{\alpha_{2}}\right)-g_{n}\left(P_{\alpha_{1}}\right)\right| \leqq a d^{\gamma}\left(P_{k_{1}}, P_{k_{2}}\right) .
$$

To extend the definition of $f_{0}$ to the entire $Q$, let $P \in Q$ and $\left\{P_{i}\right\} \subset\left\{P_{\alpha}\right\}$ be an arbitrary sequence which converges to $P$ and define $f_{0}(P)=\lim _{i \rightarrow \infty} f_{0}\left(P_{i}\right)$. The limit exists by Cauchy criterion for $\left|f_{0}\left(P_{i_{2}}\right)-f_{0}\left(P_{i_{1}}\right)\right| \leqq \operatorname{ad}^{\gamma}\left(P_{i_{1}}, P_{i_{2}}\right)$ by (6.2) and $\lim _{i_{1}, i_{2} \rightarrow \infty} d^{\gamma}\left(P_{i_{1}}, P_{i_{2}}\right)=0$. The definition of $f_{0}(P)$ above is unique. For if another sequence $\left\{P_{j}\right\} \subset\left\{P_{\alpha}\right\}$ also converges to $P$, then

$$
\left|\lim _{j \rightarrow \infty} f_{0}\left(P_{j}\right)-\lim _{i \rightarrow \infty} f_{0}\left(P_{i}\right)\right| \leqq \lim _{i, j \rightarrow \infty} a d^{\gamma}\left(P_{i}, P_{j}\right)=0 .
$$

The function $f_{0}(P)$ defined above is continuous on $Q$. To show this let $\left\{P_{l}\right\} \subset Q$ be an arbitrary sequence which converges to $P$, and for each $P_{l}$ let 
$\left\{P_{l, k}\right\} \subset\left\{P_{\alpha}\right\}$ be a sequence which converges to $P_{l}$, and let $\left\{P_{i}\right\} \subset\left\{P_{\alpha}\right\}$ be a sequence which converges to $P$. Then

$$
\begin{aligned}
\left|\lim _{l \rightarrow \infty} f_{0}\left(P_{l}\right)-f_{0}(P)\right| & =\left|\lim _{l \rightarrow \infty} \lim _{k \rightarrow \infty} \lim _{n \rightarrow \infty} g_{n}\left(P_{l, k}\right)-\lim _{i \rightarrow \infty} \lim _{n \rightarrow \infty} g_{n}\left(P_{i}\right)\right| \\
& \leqq \lim _{l, k, i \rightarrow \infty} a d^{\gamma}\left(P_{l, k}, P_{i}\right)=0 .
\end{aligned}
$$

To show that $f_{0} \in A_{\alpha}$ let $P, P^{\prime} \in Q$ and $\left\{P_{i}\right\},\left\{P_{j}\right\} \subset\left\{P_{\alpha}\right\}$ be two subsequences which converges to $P, P^{\prime}$ respectively. Then

$$
\begin{aligned}
\left|f_{0}\left(P^{\prime}\right)-f_{0}(P)\right| & =\left|\lim _{j \rightarrow \infty} \lim _{n \rightarrow \infty} g_{n}\left(P_{j}\right)-\lim _{i \rightarrow \infty} \lim _{n \rightarrow \infty} g_{n}\left(P_{i}\right)\right| \\
& \leqq \lim _{i, j \rightarrow \infty} a d^{\gamma}\left(P_{\imath}, P_{j}\right)=a d^{\gamma}\left(P, P^{\prime}\right) .
\end{aligned}
$$

Finally to show that $\lim _{n \rightarrow \infty} g_{n}(P)=f_{0}(P)$ on $Q$, let $\epsilon>0$ be given, $\left\{P_{i}\right\} \subset\left\{P_{\alpha}\right\}$ be a sequence which converges to $P, i_{0}$ be so large that $d\left(P_{i_{0}}, P\right)$ $\leqq(\epsilon / 3 a)^{1 / \gamma}$ and $N$ be such that $\left|g_{n}\left(P_{i_{0}}\right)-f_{0}\left(P_{i_{0}}\right)\right|<\epsilon / 3$ for $n \geqq N$. Then since $g_{n}, f_{0} \in A_{a}$

$$
\begin{aligned}
\left|g_{n}(P)-f_{0}(P)\right| \leqq & \left|g_{n}(P)-g_{n}\left(P_{i_{0}}\right)\right|+\left|g_{n}\left(P_{i_{0}}\right)-f_{0}\left(P_{i_{0}}\right)\right| \\
& +\left|f_{0}\left(P_{i_{0}}\right)-f_{0}(P)\right| \\
\leqq & a d^{\gamma}\left(P_{i_{0}}, P\right)+\frac{\epsilon}{3}+a d^{\gamma}\left(P_{i_{0}}, P\right) \leqq \epsilon \quad \text { for } n \geqq N
\end{aligned}
$$

and $\lim _{n \rightarrow \infty} g_{n}(P)=f_{0}(P)$. This completes the proof that the subsequence $\left\{g_{n}\right\} \subset\left\{f_{n}\right\}$ converges to $f_{0} \in A_{a}$.

Corollary. Let $J \in \mathcal{g}$ be defined by st restriction points $\left(x_{1}, y_{1}\right), \cdots,\left(x_{s}, y_{t}\right)$ and $a$ set $G$ closed in the usual topology of $R_{s}$. Then $J \cap A_{a}$ is compact in itself.

Proof. Since a set closed in the usual topology of a Euclidean space is a Lebesgue measurable set, $J$ is indeed an interval. Let a sequence $\left\{f_{n}\right\} \subset J \cap A_{a}$ be given. By Lemma 5 , there exists a subsequence $\left\{g_{n}\right\}$ of $\left\{f_{n}\right\}$ which converges to an element $f_{0}$ of $A_{a}$. To prove the corollary it remains to show that $f_{0} \in J$ also. From the pointwise convergence of $\left\{g_{n}\right\}$ to $f_{0}$ on $Q$ and in particular on the restriction points, it follows that

$$
\lim _{n \rightarrow \infty}\left[g_{n}\left(x_{1}, y_{1}\right), \cdots, g_{n}\left(x_{s}, y_{t}\right)\right]=\left[f_{0}\left(x_{1}, y_{1}\right), \cdots, f_{0}\left(x_{s}, y_{t}\right)\right]
$$

in the usual topology of $R_{s t}$. Then since $\left\{f_{n}\right\} \subset J,\left[g_{n}\left(x_{1}, y_{1}\right), \cdots, g_{n}\left(x_{s}, y_{t}\right)\right]$ $\in G$ and since $G$ is closed, $\left[f_{0}\left(x_{1}, y_{1}\right), \cdots, f_{0}\left(x_{s}, y_{t}\right)\right] \in G$ and $f_{0} \in J$.

7. Lemma 6. Let $I \in \mathcal{J}$ be defined by st restriction points $\left(x_{1}, y_{1}\right), \cdots,\left(x_{8}, y_{t}\right)$ and a Lebesgue measurable subset $E$ of $R_{s t}$ as restricting set. Then for any $\epsilon>0$ 
there exists a closed set $G \subset E$ such that the interval $J$ defined by the same st restriction points and with $G$ as restricting set satisfies $m(I-J)<\epsilon$.

Proof. If $E$ is a Legesgue measurable set in $R_{s t}$, then for any $\epsilon_{0}>0$ there exists a closed set $G \subset E$ such that (Leb) $m(E-G)<\epsilon_{0}$. Let

$$
\epsilon_{0}=\epsilon\left\{\pi^{s t}\left[x_{1}\left(x_{2}-x_{1}\right), \cdots,\left(x_{8}-x_{s-1}\right)\right]^{t}\left[y_{1}\left(y_{2}-y_{1}\right) \cdots\left(y_{t}-y_{t-1}\right)^{s}\right\}\right]^{1 / 2} .
$$

The interval $I-J$ is defined by the same st restriction points and with the measurable set $E-G$ as restriction set and hence by (2.2), (2.3)

$$
\begin{aligned}
m(J-I) & =\int \underset{E-G}{(s t)} \int W\left\{x_{1}, \cdots, x_{s}, y_{1}, \cdots, y_{t}\right\} d u_{11} \cdots d u_{s t} \\
& \leqq(\operatorname{Leb}) m(E-G) \sup _{E-G} W\left\{x_{1}, \cdots, x_{s}, y_{1}, \cdots, y_{t}\right\} \leqq \epsilon .
\end{aligned}
$$

\section{Countable additivity of $m(I)$.}

THEOREM II. The set function $m(I)$ is countably additive on $\mathcal{g}$, i.e., if a sequence of intervals $\left\{I_{n}\right\} \subset \mathcal{J}$ is such that $I_{i} \cap I_{j}=\varnothing$ for $i \neq j$ and $I=\cup_{n=1}^{\infty} I_{n} \in \mathcal{J}$, then $m(I)=\sum_{i=1}^{\infty} m\left(I_{n}\right)$.

Proof. (1) Since $\mathcal{J}$ is a field $J_{n}=\left(I-\bigcup_{j=1}^{n} I_{n}\right) \in \mathcal{J}$. It can be seen readily that $\bigcap_{n=1}^{\infty} J_{n}=\varnothing$. Then since $\left\{J_{n}\right\}$ is a monotonically decreasing sequence, $\lim _{n \rightarrow \infty} J_{n}=\bigcap_{n=1}^{\infty} J_{n}=\varnothing$. From the fact that $\left(\bigcup_{j=1}^{n} I_{j}\right) \cap J_{n}=\varnothing, I=\left(\bigcup_{j=1}^{n} I_{j}\right)$ $\cup J_{n}$ and $m$ is finite additive,

$$
m(I)=m\left(\bigcup_{j=1}^{n} I_{j}\right)+m\left(J_{n}\right)=\sum_{j=1}^{n} m\left(I_{j}\right)+m\left(J_{n}\right) \quad \text { for all } n .
$$

For the proof of the countable additivity of $m$ we only have to show that $\lim _{n \rightarrow \infty} m\left(J_{n}\right)=0$, i.e. for given $\epsilon>0$, there exists an integer $n_{0}$ such that $m\left(J_{n}\right)<\epsilon$ for $n \geqq n_{0}$.

(2) Let $J_{n}$ be defined by $s_{n} t_{n}$ restriction points $\left(x_{1}^{n}, y_{1}^{n}\right), \cdots,\left(x_{s_{n}}^{n}, y_{t_{n}}^{n}\right)$ with a restricting set $E_{n}$ which is a Lebesgue measurable subset of $R_{\boldsymbol{s}_{n} t_{n}}$. By Lemma 6 , there exists a closed set $G_{n} \subset E_{n}$ such that the interval $K_{n}$ defined by the same $s_{n} t_{n}$ restriction points and the restricting set $G_{n}$ satisfies $m\left(J_{n}-K_{n}\right)$ $<\epsilon / 2^{n+1}$. Let $L_{n}=\bigcap_{j=1}^{n} K_{j}$, and use the closed set which is the intersection of the restricting sets of $K_{1}, \cdots, K_{n}$ as its restricting set (the restricting sets all being raised to the same Euclidean dimensionality). Since $L_{n}, J_{n}-L_{n}$ are intervals, $L_{n} \subset K_{n} \subset J_{n}$ and $m$ is finitely additive

$$
m\left(J_{n}\right)=m\left(J_{n}-L_{n}\right)+m\left(L_{n}\right) .
$$

It can be seen readily that $J_{n}-L_{n}=J_{n}-\bigcap_{j=1}^{n} K_{j} \subset \bigcup_{j=1}^{n}\left(J_{j}-K_{j}\right)$, and hence from the finite additivity of $m$

$$
m\left(J_{n}-L_{n}\right) \leqq \sum_{j=1}^{n} m\left(J_{j}-K_{j}\right)<\sum_{j=1}^{n} \frac{\epsilon}{2^{j+1}}<\frac{\epsilon}{2} \quad \text { for all } n .
$$


(3) It remains to show that there exists an integer $n_{0}$ such that $m\left(L_{n}\right)$ $<\epsilon / 2$ for $n \geqq n_{0}$ by means of Theorem I. Let $a>0$ be so chosen that for any interval disjoint from $A_{a}$, its measure is less than $\epsilon / 2$. To show that $L_{n} \cap A_{a}$ $=\varnothing$ for $n \geqq n_{0}$ for some integer $n_{0}$. Let $M_{n}=L_{n} \cap A_{a}$. Since $\left\{L_{n}\right\}$ is monotonically decreasing, so is $\left\{M_{n}\right\}$ and $\lim _{n \rightarrow \infty} M_{n}=\bigcap_{n=1}^{\infty} M_{n} \subset \bigcap_{n=1}^{\infty} J_{n}=\varnothing$. We claim that there exists an integer $n_{0}$ such that $M_{n}=\varnothing$ for $n \geqq n_{0}$. Assume the contrary, then since $\left\{M_{n}\right\}$ is monotonically decreasing none of $\left\{M_{n}\right\}$ is empty. Let $f_{n} \in M_{n}$ and consider the sequence $\left\{f_{n}\right\}$. By Lemma 5 , there exists a subsequence $\left\{f_{n_{k}}\right\} \subset\left\{f_{n}\right\}$ which converges to an element $f_{0}$ of $A_{a}$. To show that $f_{0} \in M_{n_{k}}$ for all $n_{k}$, let $n_{k_{0}}$ be arbitrarily fixed. Since $\left\{M_{n_{k}}\right\}$ is monotonically decreasing $f_{n_{k}} \in M_{n_{k_{0}}}$ for $n_{k} \geqq n_{k_{0}}$. Then by the corollary to Lemma 5 , there exists a subsequence $\left\{f_{n_{k}}\right\} \subset\left\{f_{n_{k}}\right\}$ which converges to an element $f_{0}^{*}$ of $M_{n_{k_{0}}}$. For any $P \in Q$, the sequence of numbers $\left\{f_{n_{k_{l}}}(P)\right\}$ being a subsequence of $\left\{f_{n_{k}}(P)\right\}$ converges to $f_{0}(P)$. Thus $f_{0}^{*}(P)=f_{0}(P)$ on $Q$, and $f_{0} \in M_{n_{k_{0}}}$. From the arbitrariness of the choice of $n_{k_{0}}, f_{0} \in M_{n_{k}}$ for all $n_{k}$. Now since $f_{0}$ belongs to infinitely many $M_{n}, f_{0} \in \lim \sup _{n \rightarrow \infty} M_{n}=\lim _{n \rightarrow \infty} M_{n}=\varnothing$. This is a contradiction. Therefore there must exist an integer $n_{0}$ such that $M_{n}=\varnothing$ for all $n \geqq n_{0}$. Then by Theorem I $m\left(L_{n}\right)<\epsilon / 2$ for $n \geqq n_{0}$, and by (8.2), (8.3), $m\left(J_{n}\right)<\epsilon$ for $n \geqq n_{0}$. This proves the countable additivity of $m$.

\section{BiBLIOGRAPHY}

1. R. H. Cameron, Integration in function spaces, Unpublished lecture notes.

2. T. Kitagawa, Analysis of variance applied to function spaces, Mem. Fac. Sci. Kyūsyū Univ. Ser. A vol. VI no. 1 (1951) pp. 41-53, esp. pp. 44-45.

3. N. Wiener, Generalized harmonic analysis, Acta Math. vol. 55 (1930) pp. 117-258, esp. pp. $214-234$.

4. T. Hida, On the uniform continuity of the Wiener process with a multidimensional parameter, Nagoya Math. J. vol. 13 (1958) pp. 53-61.

Massachusetts Institute of Technology, Cambridge, Massachusetts 